\title{
On the neutrino mass spectrum and neutrino mixing from oscillation data
}

\author{
S.M. Bilenky ${ }^{\mathrm{a}}$, C. Giunti ${ }^{\mathrm{b}}$ and W. Grimus ${ }^{\mathrm{c}}$

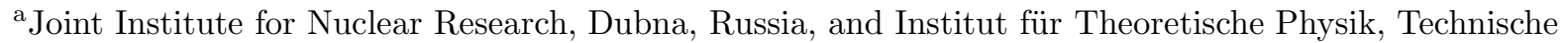 \\ Universität Munchen, D-85748 Garching, Germany \\ ${ }^{\mathrm{b}} \mathrm{INFN}$, Sezione di Torino, and Dipartimento di Fisica Teorica, Via P. Giuria 1, I-10125 Torino, Italy
}

${ }^{\mathrm{c}}$ Institute for Theoretical Physics, University of Vienna, Boltzmanngasse 5, A-1090 Vienna, Austria

Two schemes of mixing of four massive neutrinos with two couples of close neutrino masses separated by a gap of the order of $1 \mathrm{eV}$ can accommodate solar, atmospheric and LSND neutrino oscillation data. It is shown that long-baseline $\bar{\nu}_{e} \rightarrow \bar{\nu}_{e}$ and $\nu_{\mu} \rightarrow \nu_{e}$ transitions are strongly suppressed in these schemes. The scheme of mixing of three neutrino masses with a mass hierarchy that can describe solar and atmospheric neutrino data is also discussed. It is shown that in this scheme the effective Majorana mass $|\langle m\rangle|$ that characterizes the matrix element of neutrinoless double- $\beta$ decay is less than $\sim 10^{-2} \mathrm{eV}$.

Preprint TUM-HEP-327/98, SFB 375-307, UWThPh-1998-50, DFTT 52/98, hep-ph/9809368.

Talk presented by S.M. Bilenky at Neutrino '98, Takayama, Japan, June 1998.

\section{Introduction}

The conference Neutrino '98 is a very important event in neutrino physics. At this conference the Super-Kamiokande collaboration [1] presented the results of 535 days of measurement of the atmospheric neutrino fluxes which provide an impressive evidence for neutrino oscillations.

We discuss here which indications on the neutrino mass spectrum and on neutrino mixing can be obtained from the results of SuperKamiokande and all other neutrino oscillation experiments. We will discuss also possible consequences for future experiments that can be inferred from the analysis of the existing data.

In accordance with the neutrino oscillation hypothesis (see [2]) the left-handed flavor neutrino fields $\nu_{e L}, \nu_{\mu L}$ and $\nu_{\tau L}$ are linear combinations of the left-handed components of the (Dirac or Majorana) massive neutrino fields $\nu_{i}$ :

$\nu_{\alpha L}=\sum_{i} U_{\alpha k} \nu_{i L}$

In the LEP experiments on the measurement of the invisible width of the $Z$-boson it was proved that only three flavor neutrinos exist in nature (see [3]). The number of massive neutrinos can be, however, bigger than three (see [2]).
If the total lepton number $L=L_{e}+L_{\mu}+L_{\tau}$ is conserved, the neutrinos $\nu_{i}$ are Dirac particles and the number of massive neutrinos is equal to three.

If the total lepton number is not conserved, the neutrinos $\nu_{i}$ are massive Majorana particles $\left(\nu_{i}=\right.$ $\nu_{i}^{c} \equiv \mathcal{C}{\overline{\nu_{i}}}^{T}$, where $\mathcal{C}$ is the charge-conjugation matrix). In the general case, the number of Majorana fields $\nu_{i}$ is $n=3+m$, where $m$ is the number of right-handed fields $\nu_{a R}$ that enter in the neutrino mass term. We have in this case

$\nu_{\alpha L}=\sum_{i=1}^{n} U_{\alpha i} \nu_{i L}, \quad\left(\nu_{a R}\right)^{c}=\sum_{i=1}^{n} U_{a i} \nu_{i L}$,

where $U$ is a $n \times n$ unitary mixing matrix.

Two possible options are usually discussed in the Majorana case:

1. The see-saw option [四. If the total lepton number is violated by the right-handed Majorana mass term at an energy scale much larger than the electroweak scale, the Majorana mass spectrum is composed of three light masses $m_{i}(i=1,2,3)$ and three very heavy masses $M_{i}(i=1,2,3)$ that characterize the scale of lepton number violation. 
The light neutrino masses are given by the see-saw formula

$m_{i} \sim \frac{\left(m_{i}^{F}\right)^{2}}{M_{i}} \ll m_{i}^{F} \quad(i=1,2,3)$.

where $m_{i}^{F}$ is the mass of the charged lepton or up-quark in the $i^{\text {th }}$ generation. The seesaw mechanism provides a plausible explanation for the smallness of neutrino masses with respect to the masses of all other fundamental fermions.

2. The sterile neutrino option. If more than three Majorana mass terms are small, then there are light sterile neutrinos. In this case active neutrinos $\nu_{e}, \nu_{\mu}$ and $\nu_{\tau}$ can transform into sterile states $\nu_{a}$ that are quanta of right-handed fields $\nu_{a R}$. Notice that sterile neutrinos can be obtained in the framework of the see-saw mechanism with some additional assumptions ("singular see-saw" [5]).

From the analysis of the data of atmospheric neutrino experiments it follows that $\Delta m_{\text {atm }}^{2} \sim 2 \times$ $10^{-3} \mathrm{eV}^{2}$ [1], where $\Delta m^{2}$ is the difference between the squares of neutrino masses. Another scale of $\Delta m^{2}$ was obtained [6,7] from the analysis of the data of all solar neutrino experiments: $\Delta m_{\text {sun }}^{2} \sim$ $10^{-5} \mathrm{eV}^{2}$ (MSW [8]) or $\Delta m_{\text {sun }}^{2} \sim 10^{-10} \mathrm{eV}^{2}$ (vacuum oscillations). Finally, indications in favor of $\bar{\nu}_{\mu} \rightarrow \bar{\nu}_{e}$ and $\nu_{\mu} \rightarrow \nu_{e}$ oscillations with a third scale of $\Delta m^{2}, \Delta m_{\text {LSND }}^{2} \sim 1 \mathrm{eV}^{2}$, were obtained in the accelerator LSND experiment [9].

All these indications in favor of neutrino masses and mixing will be checked by future solar, longbaseline and short-baseline neutrino oscillation experiments (see these Proceedings).

We will consider two possible scenarios:

1. All three indications in favor of neutrino oscillations are confirmed.

2. Only the indications of solar and atmospheric neutrino experiments are confirmed.

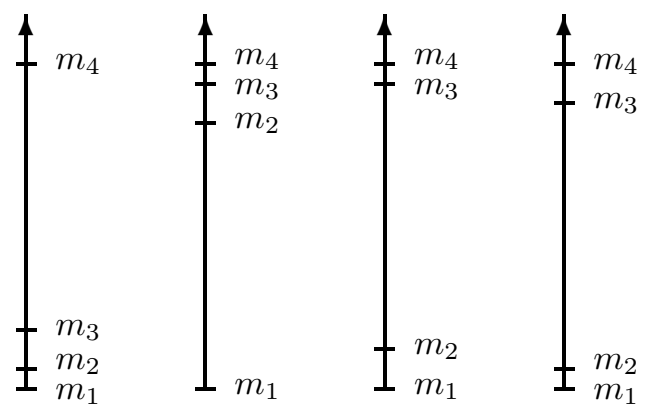

(I)

(II)

(IIIA)

(IIIB)

Figure 1

\section{Four massive neutrinos}

At least four massive neutrinos are needed in order to have three different scales of $\Delta m^{2}$ 14.57. The three types of neutrino mass spectra that can accommodate the solar, atmospheric and LSND scales of $\Delta m^{2}$ are shown in Fig. 1. In all these spectra there are two groups of close masses separated by a gap of the order of $1 \mathrm{eV}$ which gives $\Delta m_{41}^{2} \equiv m_{4}^{2}-m_{1}^{2} \simeq \Delta m_{\mathrm{LSND}}^{2}$.

Only the largest mass-squared difference $\Delta m_{41}^{2}$ is relevant for the oscillations in short-baseline (SBL) experiments and the SBL transition probabilities have the same dependence on the parameter $\Delta m_{41}^{2} L / 2 p$ ( $L$ is the source-detector distance and $p$ is the neutrino momentum) as the standard two-neutrino probabilities 11]:

$$
\begin{aligned}
P_{\nu_{\alpha} \rightarrow \nu_{\beta}} & =\frac{1}{2} A_{\alpha ; \beta}\left(1-\cos \frac{\Delta m_{41}^{2} L}{2 p}\right), \\
P_{\nu_{\alpha} \rightarrow \nu_{\alpha}} & =1-\frac{1}{2} B_{\alpha ; \alpha}\left(1-\cos \frac{\Delta m_{41}^{2} L}{2 p}\right) .
\end{aligned}
$$

The oscillation amplitudes $A_{\alpha ; \beta}$ and $B_{\alpha ; \alpha}$ depend on the elements on the mixing matrix $U$ and on the form of the neutrino mass spectrum:

$$
\begin{aligned}
& A_{\alpha ; \beta}=4\left|\sum_{i} U_{\beta i} U_{\alpha i}^{*}\right|^{2}, \\
& B_{\alpha ; \alpha}=4\left(\sum_{i}\left|U_{\alpha i}\right|^{2}\right)\left(1-\sum_{i}\left|U_{\alpha i}\right|^{2}\right),
\end{aligned}
$$

where the index $i$ runs over the indices of the first 
or (because of the unitarity of $U$ ) second group of neutrino masses.

The results of SBL reactor $\bar{\nu}_{e}$ and accelerator $\nu_{\mu}$ disappearance experiments in which no oscillations were found imply that $B_{\alpha ; \alpha} \leq B_{\alpha ; \alpha}^{0}$ for $\alpha=e, \mu$. The upper bounds $B_{\alpha ; \alpha}^{0}$ are given by the exclusion curves obtained from the data of SBL disappearance experiments and depend on the value of $\Delta m_{41}^{2}$. Using Eq.(7), these upper bounds imply the following constraints for the quantities $\sum_{i}\left|U_{\alpha i}\right|^{2}(\alpha=e, \mu)$ :

$\sum_{i}\left|U_{\alpha i}\right|^{2} \leq a_{\alpha}^{0} \quad$ or $\quad \sum_{i}\left|U_{\alpha i}\right|^{2} \geq 1-a_{\alpha}^{0}$,

where

$a_{\alpha}^{0}=\frac{1}{2}\left(1-\sqrt{1-B_{\alpha ; \alpha}^{0}}\right)$.

The most stringent values of $a_{e}^{0}$ and $a_{\mu}^{0}$ can be obtained from the results of the Bugey reactor experiment 15] and of the CDHS and CCFR accelerator experiments 16].

We have considered the range $10^{-1} \leq \Delta m_{41}^{2} \leq$ $10^{3} \mathrm{eV}^{2}$. In this range $a_{e}^{0} \lesssim 4 \times 10^{-2}$ and $a_{\mu}^{0} \lesssim 2 \times 10^{-1}$ for $\Delta m_{41}^{2} \gtrsim 0.3 \mathrm{eV}^{2}$ (see Fig. 1 of Ref. [17). Thus, from the results of disappearance experiments it follows that $\sum_{i}\left|U_{e i}\right|^{2}$ and $\sum_{i}\left|U_{\mu i}\right|^{2}$ can be either small or large (close to one).

From the four possible sets of values of the quantities $\sum_{i}\left|U_{e i}\right|^{2}$ and $\sum_{i}\left|U_{\mu i}\right|^{2}$ (small-small, small-large, large-small and large-large), for each neutrino mass spectrum only one set is compatible with the results of solar and atmospheric neutrino experiments 11, 12. In the case of spectra I and II we have

$\left|U_{e k}\right|^{2} \leq a_{e}^{0} \quad$ and $\quad\left|U_{\mu k}\right|^{2} \leq a_{\mu}^{0}$,

with $k=4$ for spectra I and $k=1$ for spectra II. In the case of spectrum IIIA we have

$$
\sum_{i=1,2}\left|U_{e i}\right|^{2} \leq a_{e}^{0} \text { and } \sum_{i=1,2}\left|U_{\mu i}\right|^{2} \geq 1-a_{\mu}^{0}
$$

whereas in the case of spectrum IIIB we have

$$
\sum_{i=3,4}\left|U_{e i}\right|^{2} \leq a_{e}^{0} \text { and } \sum_{i=3,4}\left|U_{\mu i}\right|^{2} \geq 1-a_{\mu}^{0} .
$$

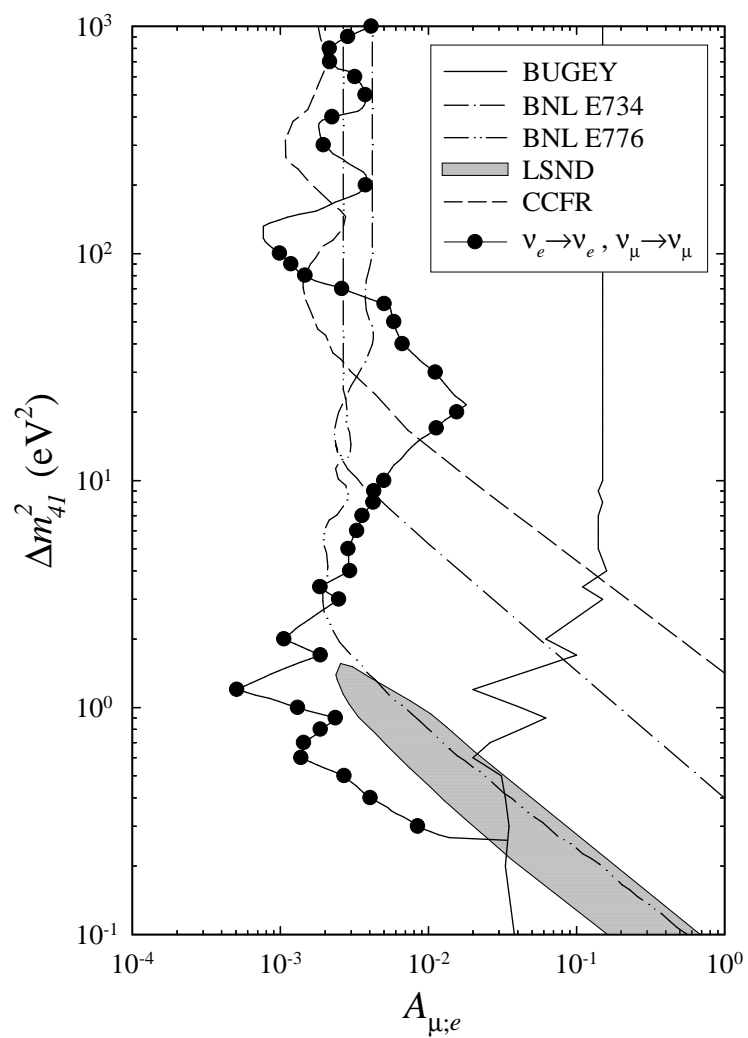

Figure日

In the case of spectra I and II, $\nu_{\mu} \rightarrow \nu_{e}$ transitions in SBL experiments are strongly suppressed. Indeed, we have

$A_{e ; \mu} \leq 4\left|U_{e k}\right|^{2}\left|U_{\mu k}\right|^{2} \leq 4 a_{e}^{0} a_{\mu}^{0}$.

In Fig. 2 the upper bound (13) is compared with the latest LSND-allowed region (at 90\% CL). Figure 2 shows that the spectra of type I and II (that include also the hierarchical spectrum) are disfavored by the result of the LSND experiment (they are compatible with the results of the LSND experiment only in the narrow region of $\Delta m_{41}^{2}$ around $0.2-0.3 \mathrm{eV}^{2}$, where there is no information on $B_{\mu ; \mu}$ ).

On the other hand, there is no incompatibility of the spectra IIIA and IIIB with the results of the LSND experiment and we conclude that these two spectra are favored by the existing data.

We discuss now some consequences for future experiments that can be inferred from the 
schemes IIIA and IIIB. Let us discuss first the possibilities for the effective neutrino mass $m\left({ }^{3} \mathrm{H}\right)$ measured in tritium $\beta$-decay experiments and for the effective Majorana mass $|\langle m\rangle| \equiv\left|\sum_{k} U_{e k}^{2} m_{k}\right|$ measured in neutrinoless double- $\beta$ decay experiments.

In scheme IIIA we have

$m\left({ }^{3} \mathrm{H}\right) \simeq m_{4}, \quad|\langle m\rangle| \leq m_{4}$,

whereas in scheme IIIB

$m\left({ }^{3} \mathrm{H}\right) \leq a_{e}^{0} m_{4} \ll m_{4}$,

$|\langle m\rangle| \leq a_{e}^{0} m_{4} \ll m_{4}$.

Therefore, if the scheme A is realized in nature, tritium $\beta$-decay experiments experiments and neutrinoless double- $\beta$ decay experiments have a possibility to see the effects of the relatively large neutrino mass $m_{4} \simeq \sqrt{\Delta m_{\mathrm{LSND}}^{2}}$.

Let us consider now neutrino transitions in long-baseline (LBL) experiments. In the scheme IIIA the LBL transition probabilities are given by 13

$$
\begin{aligned}
P_{\nu_{\alpha} \rightarrow \nu_{\beta}}^{\mathrm{LBL}}= & \left|\sum_{k=1,2} U_{\alpha k}^{*} e^{-i \frac{\Delta m_{k 1}^{2} L}{2 E}} U_{\beta k}\right|^{2} \\
& +\left|\sum_{j=3,4} U_{\alpha j}^{*} U_{\beta j}\right|^{2} .
\end{aligned}
$$

The transition probabilities in the scheme IIIB can be obtained from (16) with the change 1,2 3,4 . The inequalities (11) and (12) imply strong constraints on the probabilities of $\bar{\nu}_{e} \rightarrow \bar{\nu}_{e}$ and $\nu_{\mu} \rightarrow \nu_{e}$ transitions in LBL experiments [13]. Indeed, for the probability of $\bar{\nu}_{e} \rightarrow \bar{\nu}_{e}$ transitions we have

$P_{\bar{\nu}_{e} \rightarrow \bar{\nu}_{e}}^{\mathrm{LBL}} \geq\left(\sum_{j=3,4}\left|U_{e j}\right|^{2}\right)^{2} \geq\left(1-a_{e}^{0}\right)^{2}$

in scheme IIIA and

$P_{\bar{\nu}_{e} \rightarrow \bar{\nu}_{e}}^{\mathrm{LBL}} \geq\left(\sum_{k=1,2}\left|U_{e j}\right|^{2}\right)^{2} \geq\left(1-a_{e}^{0}\right)^{2}$

in scheme IIIB. Hence, in both schemes $P_{\bar{\nu}_{e} \rightarrow \bar{\nu}_{e}}^{\mathrm{LBL}}$ is close to one and the LBL probability of transi-

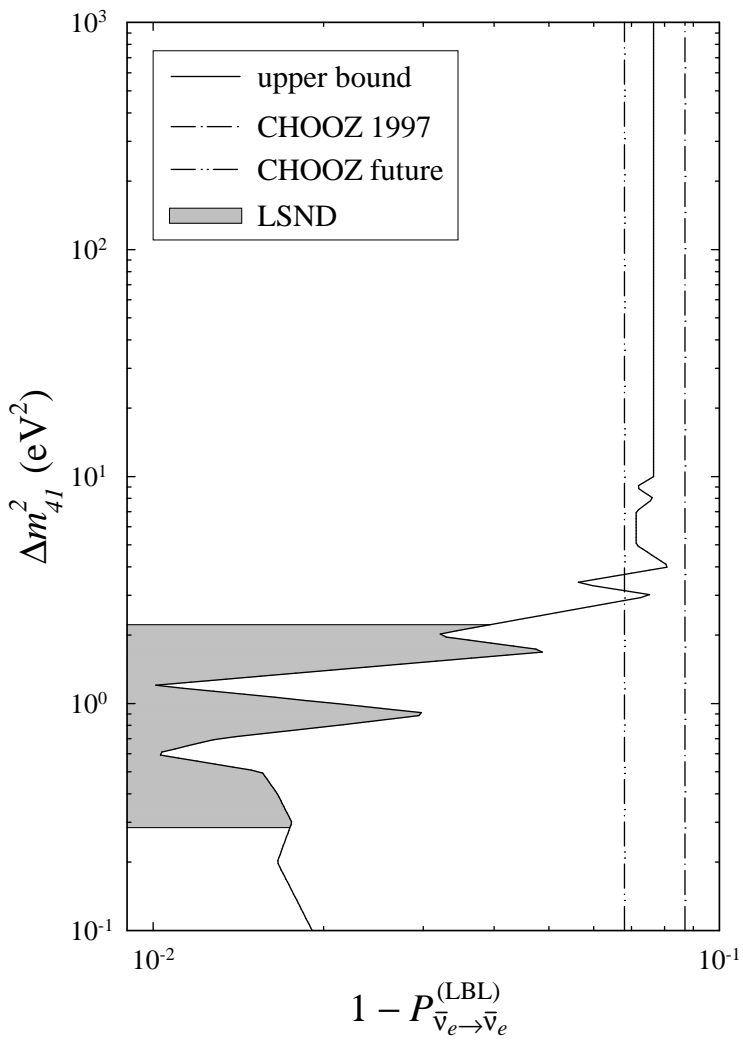

Figure 3

tions of $\bar{\nu}_{e}$ into any other state is small. Indeed, in both schemes we have

$1-P_{\bar{\nu}_{e} \rightarrow \bar{\nu}_{e}}^{\mathrm{LBL}} \leq a_{e}^{0}\left(2-a_{e}^{0}\right)$.

This limit is shown by the solid line in Fig. 3 . The upper bound for the transition probability $1-P_{\bar{\nu}_{e} \rightarrow \bar{\nu}_{e}}^{\mathrm{LBL}}$ obtained in the $\mathrm{CHOOZ}$ experiment 18$]$ (dash-dotted line) and the final sensitivity of the $\mathrm{CHOOZ}$ experiment (dash-dotdotted line) are also shown. It can be seen that for $\Delta m_{41}^{2} \lesssim 1 \mathrm{eV}^{2}$ the upper bound (19) for $1-P_{\bar{\nu}_{e} \rightarrow \bar{\nu}_{e}}^{\mathrm{LBL}}$ is much smaller than the upper bound reached in the $\mathrm{CHOOZ}$ experiment and than the final sensitivity of the experiment.

\section{Three massive neutrinos}

If the results of the LSND experiment will not be confirmed by future experiments, the most plausible scheme is the one with mixing 
of three massive neutrinos and a mass hierarchy 19,20,17]:

$m_{1} \ll m_{2} \ll m_{3}$.

The effective Majorana mass that characterize the matrix element of neutrinoless double- $\beta$ decay is given in this case by [20]

$|\langle m\rangle| \simeq\left|U_{e 3}\right|^{2} \sqrt{\Delta m_{31}^{2}}$.

The results of reactor neutrino experiments imply the upper bound $\left|U_{e 3}\right|^{2} \leq a_{e}^{0}$, with $a_{e}^{0}$ given in Eq.(9). Therefore the effective Majorana mass is bounded by

$|\langle m\rangle| \lesssim a_{e}^{0} \sqrt{\Delta m_{31}^{2}}$.

The value of this upper bound as a function $\Delta m_{31}^{2}$ obtained from 90\% CL exclusion plots of the Bugey [15] and CHOOZ 18] experiments is presented in Fig. (the solid and dashed line, respectively). The region on the right of the thick straight solid line is forbidden by the unitarity bound $|\langle m\rangle| \leq \sqrt{\Delta m_{31}^{2}}$.

Also the results of the Super-Kamiokande atmospheric neutrino experiment imply an upper bound for $\left|U_{e 3}\right|^{2}$. The shadowed region in Fig. 4 shows the region allowed by Super-Kamiokande results at $90 \% \mathrm{CL}$ that we have obtained using the results of three-neutrino analysis performed by Yasuda 21].

Figure 1 shows that the results of the SuperKamiokande and $\mathrm{CHOOZ}$ experiments imply that $|\langle m\rangle| \lesssim 10^{-2} \mathrm{eV}$.

The observation of neutrinoless double- $\beta$ decay with a probability that corresponds to a value of $|\langle m\rangle|$ significantly larger than $10^{-2} \mathrm{eV}$ would mean that the masses of three neutrinos do not have a hierarchical pattern and/or exotic mechanisms (right-handed currents, supersymmetry with violation of R-parity, ..., see [22]) are responsible for the process.

Let us notice that from the results of the Heidelberg-Moscow ${ }^{76} \mathrm{Ge}$ experiment [23] it follows that $|\langle m\rangle| \lesssim 0.5-1.5 \mathrm{eV}$. The next generation of neutrinoless double- $\beta$ experiments will reach $|\langle m\rangle| \simeq 10^{-1} \mathrm{eV}$ 24]. Possibilities to reach $|\langle m\rangle| \simeq 10^{-2} \mathrm{eV}$ are under discussion 24].

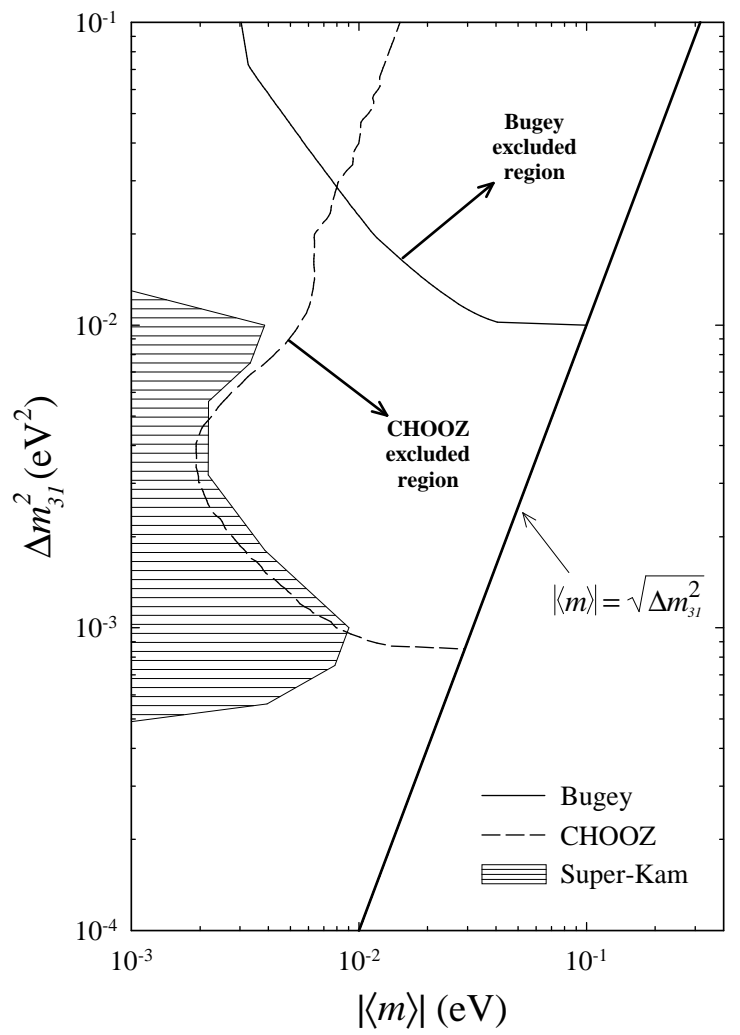

Figure 6

\section{Conclusions}

In conclusion, the neutrino mass spectrum and the structure of the neutrino mixing matrix depend crucially on the confirmation of the results of the LSND experiment. If these results will be confirmed we need (at least) four massive neutrinos with a mass spectrum of type IIIA or IIIB (see Fig. 国). If the results of the LSND experiment will not be confirmed, the most plausible scenario is the one with three massive neutrinos and a mass hierarchy. The investigation of the nature of massive neutrinos (Dirac or Majorana?) will require in this case to reach a sensitivity of $|\langle m\rangle| \lesssim 10^{-2} \mathrm{eV}$ in searching for neutrinoless double- $\beta$ decay.

\section{REFERENCES}


1. T. Kajita, Talk presented at Neutrino '98, these Proceedings; Y. Fukuda et al., hepex/9807003.

2. S.M. Bilenky and B. Pontecorvo, Phys. Rep. 41, 225 (1978); S.M. Bilenky and S.T. Petcov, Rev. Mod. Phys. 59, 671 (1987).

3. C. Caso et al, Eur. Phys. J. C 3, 1 (1998).

4. M. Gell-Mann, P. Ramond, and R. Slansky, in Supergravity, ed. F. van Nieuwenhuizen and D. Freedman (North Holland, Amsterdam, 1979), p.315; T. Yanagida, Proc. of the Workshop on Unified Theory and the Baryon Number of the Universe, KEK, Japan, 1979; R.N. Mohapatra and G. Senjanović, Phys. Rev. Lett. 44, 912 (1980).

5. E.J. Chun, C.W. Kim and U.W. Lee, hepph/9802209.

6. N. Hata and P. Langacker, Phys. Rev. D 56, 6107 (1997).

7. G.L. Fogli, E. Lisi and D. Montanino, hepph/9709473.

8. S.P. Mikheyev and A.Yu. Smirnov, Yad. Fiz. 42, 1441 (1985) [Sov. J. Nucl. Phys. 42, 913 (1985)]; Il Nuovo Cimento C 9, 17 (1986); L. Wolfenstein, Phys. Rev. D 17, 2369 (1978); ibid. 20, 2634 (1979).

9. C. Athanassopoulos et al., Phys. Rev. Lett. 77, 3082 (1996); D.H. White, Talk presented at Neutrino '98, these Proceedings.

10. J.T. Peltoniemi and J.W.F. Valle, Nucl. Phys. B 406, 409 (1993); D.O. Caldwell and R.N. Mohapatra, Phys. Rev. D 48, 3259 (1993); Z. Berezhiani and R.N. Mohapatra, ibid 52, 6607 (1995); E. Ma and P. Roy, ibid 52, R4780 (1995); R. Foot and R.R. Volkas, ibid 52, 6595 (1995); S. Goswami, ibid 55, 2931 (1997); A.Yu. Smirnov and M. Tanimoto, ibid 55, 1665 (1997); J.R. Primack et al., Phys. Rev. Lett. 74, 2160 (1995); K. Benakli and A.Yu. Smirnov, ibid 79, 4314 (1997); E.J. Chun et al., Phys. Lett. B 357, 608 (1995); J.J. Gomez-Cadenas and M.C. Gonzalez-Garcia, Z. Phys. C 71, 443 (1996); E. Ma, Mod. Phys. Lett. A 11, 1893 (1996); N. Okada and O. Yasuda, Int. J. Mod. Phys. A 12, 3669 (1997); V. Barger, T.J. Weiler and K. Whisnant, hep-ph/9712495; S.C. Gibbons et al.,hep-ph/9803299; N. Gaur et al., hep-ph/9806272; V. Barger et al.,hep$\mathrm{ph} / 9806328$.

11. S.M. Bilenky, C. Giunti, C.W. Kim and S.T. Petcov, Phys. Rev. D 54, 4432 (1996).

12. S.M. Bilenky, C. Giunti and W. Grimus, Proc. of Neutrino96, Helsinki, June 1996, edited by K. Enqvist et al., p.174 (World Scientific, Singapore, 1997); Eur. Phys. J. C 1, 247 (1998).

13. S.M. Bilenky, C. Giunti and W. Grimus, Phys. Rev. D 57 (1998) 1920.

14. S.M. Bilenky, C. Giunti and W. Grimus, Phys. Rev. D 58, 033001 (1998); S.M. Bilenky, C. Giunti, W. Grimus and T. Schwetz, hep-ph/9804421.

15. B. Achkar et al., Nucl. Phys. B 434, 503 (1995).

16. F. Dydak et al., Phys. Lett. B 134, 281 (1984); I.E. Stockdale et al., Phys. Rev. Lett. 52, 1384 (1984).

17. S.M. Bilenky, A. Bottino, C. Giunti and C.W. Kim, Phys. Rev. D 54, 1881 (1996).

18. M. Apollonio et al., Phys. Lett. B 420, 397 (1998).

19. A. De Rujula et al., Nucl. Phys. B 168, 54 (1980); V. Barger and K. Whisnant, Phys. Lett. B 209, 365 (1988); S.M. Bilenky et al., ibid. 276, 223 (1992); S.M. Bilenky et al., ibid. 356, 273 (1995); K.S. Babu et al., ibid. 359, 351 (1995); H. Minakata, ibid. 356, 61 (1995); Phys. Rev. D 52, 6630 (1995); G.L. Fogli et al., ibid. 52, 5334 (1995); S. Goswami et al., Int. J. Mod. Phys. A 12, 781 (1997); S.M. Bilenky and C. Giunti, hep-ph/9802201; V. Barger et al.,hep-ph/9806387; A.J. Baltz et al., hep-ph/9806540; V. Barger et al.,hepph/9807319; Y. Nomura and T. Yanagida, hep-ph/9807325; G. Altarelli and F. Feruglio, hep-ph/9807353; E. Ma, hep-ph/9807386; N. Haba, hep-ph/9807552.

20. S.T. Petcov and A.Yu. Smirnov, Phys. Lett. B 322, 109 (1994); S.M. Bilenky et al.,Phys. Rev. D 57, 6981 (1998).

21. O. Yasuda, hep-ph/9804400.

22. R.N. Mohapatra, hep-ph/9507234; Talk presented at Neutrino '98, these Proceedings.

23. M. Günther et al., Phys. Rev. D 55, 54 (1997); Phys. Lett. B 407, 219 (1997). 
24. Talks presented by A. Morales, H. Ejiri, F. Piquemal, H.V. Klapdor-Kleingrothaus and O. Cremonesi at Neutrino '98, these Proceedings. 